\title{
BORDER REDUCTION IN EXISTENCE PROBLEMS OF HARMONIC FORMS
}

\author{
MITSURU NAKAI and LEO SARIO
}

Dedicated to Professor Kiyoshi Noshiro on his 60th birthday

1. Given an arbitrary Riemannian $n$-space $V$ let $\sigma$ be a harmonic field in the complement $V-V_{0}$ of a regular region $V_{0}$. The problem of constructing in $V$ a harmonic field $\rho$ with the property $\|\rho-\sigma\|_{r-\bar{r}_{0}}^{2}=\int_{V-\bar{r}_{0}}(\rho-\sigma) \wedge *(\rho-\sigma)<\infty$ was given a complete solution in [2]. The corresponding problem for harmonic forms $\sigma, \rho$ remains open in the general case. In the special case of locally flat spaces the construction can be carried out by replacing $\|\cdot\|$ by the point norm [3].

In the present paper we shall introduce another device that appears promising of generalization: We shall show that the border $\partial V_{0}$ can be reduced, in a sense to be specified, to an $(n-1)$-sphere. This will enable us to perform all calculations in $V_{0}$ in terms of one coordinate system.

The present paper is thus methodological in nature. However, we believe the border reduction also has some interest in its own right.

For any form $\psi=\psi_{i_{1} \ldots i_{p}} d x^{i_{1}} \wedge \cdots \wedge d x^{i_{p}}$ the point norm $|\psi|$ defined by the invariant function

$$
|\psi|^{2}=\psi_{i_{1} \ldots i_{p}} \psi^{i_{1} \ldots i_{p}}
$$

reduces to $\left(\sum \psi_{i_{1} \cdots i_{p}}^{2}\right)^{1 / 2}$ in the present case of a Euclidean metric.

A form is said to be defined in a set $E \subset V$ if it is defined in an open set containing $E$. The same is true of properties such as harmonicity that involve differentials.

2. A Riemannian space $V$ is locally flat if we can find an admissible coordinate system at each point such that the metric tensor takes the form

Received June 22, 1966.

This work was sponsored by the U. S. Army Research Office-Durham, Grant DAAROD-31-124-G742, University of California, Los Angeles. 
$g_{i j}=\delta_{i j}$ throughout the parametric neighborhood. In this case the LaplaceBeltrami operator $\Delta=d \delta+\delta d$ applied to a $p$-form $\varphi=\varphi_{i_{1} \ldots i_{p}} d x^{i_{1}} \wedge \cdots \wedge d x^{i_{p}}$ gives

$$
\Delta \varphi=\left(\Delta \varphi_{i_{1} \ldots i_{p}}\right) d x^{i_{1}} \wedge \cdots \wedge d x^{i_{p}}
$$

with

$$
\Delta \varphi_{i_{1} \ldots i_{p}}=-\left(\frac{\partial^{2}}{\left(\partial x^{1}\right)^{2}}+\cdots+\frac{\partial^{2}}{\left(\partial x^{n}\right)^{2}}\right) \varphi_{i_{1} \ldots i_{p}}
$$

Therefore a harmonic form $\varphi=\varphi_{i_{1} \ldots i_{\nu}} d x^{i_{1}} \wedge \cdots \wedge d x^{i_{y}}$ is nothing but a form whose coefficients $\varphi_{i_{3} \ldots i_{p}}$ are harmonic functions in the Euclidean coordinate system.

A Riemannian space $V$ is called hyperbolic if $V$ carries no nonconstant positive superharmonic functions, i.e., functions $v$ of class $C^{2}$ with $\Delta v \leq 0$ in $V$. Take a regular region $B$ in $V$. Then $V$ is hyperbolic if and only if there exists a continuous function $u_{\partial B}$ in $V$ such that (a) $u_{\partial B}$ is harmonic in $V-\bar{B}$, (b) $u_{\partial B} \mid B \equiv 1$, (c) $0<u_{\partial B}<1$ in $V-\bar{B}$, and (d) if $u$ is continuous in $V$ and harmonic in $V-\bar{B}$ with $u=0$ on $\bar{B}$, then $|u| \leq u_{\partial B}$ implies $u \equiv 0$.

3. Let $V_{0}$ be a regular region [4] of $V$ and let $\sigma$ be a harmonic $p$-form in the closure of $V_{\sigma}=V-\bar{V}_{0}$. Take an exhaustion [4] of $V$, say $\left\{V_{n}\right\}_{0}^{\infty}$ and let $\varphi$ be a $p$-form on $\partial V_{n}$ with tangential component $t \varphi$ and normal component $n \varphi$ on $\partial V_{n}$. For a $p$-form $\psi$ we write

$$
\varphi=\psi \text { on } \partial V_{n}
$$

if $t \varphi=t \psi$ and $n \varphi=n \psi$. This is equivalent to

$$
\varphi_{i_{1} \ldots i_{p}}=\psi_{i_{1} \ldots i_{\rho}} \text { on } \partial V_{n}
$$

for every admissible coordinate system at each point of $\partial V_{n}$. This in turn is equivalent to

$$
|\varphi-\psi|^{2}=0 \text { on } \partial V_{n}
$$

and implies

$$
|\varphi|^{2}=|\psi|^{2} \text { on } \partial V_{n}
$$

Let $\varphi$ be given in a vicinity of $\partial V_{0}$. By Duff-Spencer [1] there exists a unique harmonic $p$-form $L_{n}$, on $\bar{V}_{n}-V_{0}$ such that $L_{n} \varphi=\varphi$ on $\partial V_{0}$ and $L_{n} \varphi=0$ on $\partial V_{n}$. 
In general let $\psi$ be harmonic in a Euclidean neighborhood $U$. Then since the $\psi_{i_{1} \ldots i_{p}}$ are harmonic in $U, \psi_{i_{1} \ldots i_{p}} \psi^{i_{1} \cdots i_{p}}=\psi_{i_{1} \ldots i_{p}}^{2}$ (a single term, not summation) is subharmonic in $U$, and so is $|\psi|^{2}=\sum \psi_{i_{1} \ldots i_{1}} \psi^{i_{1} \cdots i_{p}}=\sum \psi_{i_{1} \cdots i_{p}}^{2}$.

In particular $\left|L_{n} \varphi\right|^{2}$ is subharmonic in $V_{n}-\bar{V}_{0}$, with $\left|L_{n}^{\prime} \rho\right|^{2}=|\varphi|^{2}$ on $\partial V_{0}$ and $\left|L_{n} \varphi\right|^{2}=0$ on $\partial V_{n}$. Set $M=\max _{\partial V_{0}}|\varphi|^{2}$ and let $\mu_{, r_{0}}$ be the harmonic measure of $\partial V_{0}$. Then

$$
0 \leq\left|L_{n} \varphi\right|^{2} \leq M u_{\partial r_{0}}
$$

and $\left(L_{n} \varphi\right)_{i_{1} \ldots i_{p}}$ is bounded harmonic in each Euclidean neighborhood. Therefore, there exists a continuous form $\tilde{\varphi}$ in $V-V_{0}$, harmonic in $V-\bar{V}_{0}$, such that a subsequence of $\left\{L_{n} \varphi\right\}$ converges to $\tilde{\varphi}$, i.e., each coefficient of $L_{n} \varphi$ converges to that of $\tilde{\varphi}$ in each neighborhood. Clearly

$$
0 \leq|\tilde{\varphi}|^{2} \leq M u \partial r_{0}
$$

and $\tilde{\varphi}=\varphi$ on $\partial V_{0}$.

Take another such form $\tilde{\varphi}^{\prime}$. Then of course $0 \leq\left|\tilde{\varphi}^{\prime}\right|^{2} \leq M u_{a r_{0}}$ and $\tilde{\varphi}^{\prime}=\varphi$ on $\partial V_{0}$. Thus

$$
\left|\tilde{\varphi}-\tilde{\varphi}^{\prime}\right|^{2} \leq\left(|\tilde{\varphi}|+\left|\tilde{\varphi}^{\prime}\right|\right)^{2} \leq 2|\tilde{\varphi}|^{2}+2|\tilde{\varphi}|^{2} \leq 4 M u_{\partial v_{0}} .
$$

On the other hand, $\widetilde{\varphi}-\widetilde{\varphi}^{\prime}=0$ and $\left|\tilde{\varphi}-\tilde{\varphi}^{\prime}\right|^{2}=0$ on $\partial V_{0}$. Thus by the subharmonicity of $\left|\tilde{\varphi}-\tilde{\varphi}^{\prime}\right|^{2}$ and the "vanishing property" of $u_{\partial v_{0}}$ at the ideal boundary, $\tilde{\varphi}=\tilde{\varphi}^{\prime}$. We conclude that

$$
L \varphi=\lim _{n} L_{n} \varphi \text { (i.e., }\left|L \varphi-L_{n} \varphi\right|^{2} \rightarrow 0 \text { ) }
$$

exists and satisfies
$(\alpha)$
$L\left(a \varphi_{1}+b^{\prime} \varphi_{2}\right)=a L \varphi_{1}+b L \varphi_{2}$,

$$
L \varphi=\varphi \text { on } \partial V_{0} \text {, }
$$

$$
|L \varphi|^{2} \leq\left(\max _{\partial v_{0}}|\varphi|^{2}\right) u_{\partial v_{0}} \text { on } V-V_{0}
$$$$
L^{2}=L \text {. }
$$

4. We shall now prove our contention that $V_{0}$ may be assumed to be a ball as small as we wish. To this end take an (open) ball $B$ such that $\bar{B} \subset V_{0} \subset \bar{V}_{0} \subset V_{1}$.

For a form $\varphi$ on $\vec{V}_{1}$ there exist, again by Duff-Spencer [1], unique harmonic forms $K \varphi$ and $K_{0} \varphi$ in $V_{1}$ and $V_{1}-\bar{B}$ respectively such that

$$
K \varphi=K_{0} \varphi=\varphi \text { on } \partial V_{1}
$$


and

$$
K_{0} \varphi=0 \text { on } \partial B \text {. }
$$

The operator $K \varphi$ will be used later, and for the present we only consider $K_{0} \varphi$.

We wish to find a continuous form $\bar{\sigma}$ on $V-B$, harmonic on $V-\bar{B}$, such that

$$
\bar{\sigma}=0 \text { on } \partial B
$$

and

$$
L(\bar{\sigma}-\sigma)=\bar{\sigma}-\sigma \text { on } V_{o}=V-\bar{V}_{0} \text {, }
$$

i.e., $\bar{\sigma}$ behaves like $\sigma$ near the ideal boundary of $V$.

We have only to find a form $\varphi$ on $\partial V_{1}$ and a form $\phi$ on $\partial V_{0}$ such that

$$
\begin{cases}\varphi-\sigma=L(\phi-\sigma) & \text { on } \partial V_{1} \\ \phi=K_{0} \varphi & \text { on } \partial V_{0} .\end{cases}
$$

In fact, then we put

$$
\bar{\sigma}=\left\{\begin{array}{l}
K_{0} \varphi \text { in } \bar{V}_{1}-B, \\
L_{\phi}+\sigma-L_{\sigma} \text { in } \bar{V}_{\sigma} .
\end{array}\right.
$$

It is well defined since on $\partial V_{0}, K_{0} \varphi=\phi=L \phi=L \phi+\sigma-L \sigma$, and on $\partial V_{1}, K_{0} \varphi=$ $\varphi=L \phi+\sigma-L \sigma$, and by Duff-Spencer [1], $K_{0} \varphi=L \phi-\sigma-L_{\sigma}$ in $\left(\bar{V}_{1}-B\right) \cap \bar{V}_{0}$.

Now $\bar{\sigma}$ is harmonic in $V-\bar{B}, \bar{\sigma}=0$ on $\partial B$, and $L(\bar{\sigma}-\sigma)=L^{2} \phi+L \sigma-L^{2} \sigma-L \sigma=$ $L(\phi-\sigma)=\bar{\sigma}-\sigma$ in $\bar{V}_{o}$.

Clearly solving (10) is equivalent to finding a form $\varphi$ on $\partial V_{1}$ such that

$$
\varphi-\sigma=L\left(K_{0} \varphi-\sigma\right) \text { on } \partial V_{1} \text {. }
$$

This is rewritten in the form

$$
\left(I-L K_{0}\right) \varphi=\sigma_{0} \text { on } \partial V_{1}
$$

with the identity operator $I$ and $\sigma_{0}=\sigma-L \sigma$. Since $\left(L K_{0}\right)^{n} \sigma_{0}$ is defined on $\partial V_{1}$, we are led to the Neumann series

$$
\varphi=\sum_{n=0}^{\infty}\left(L K_{0}\right)^{n} \sigma_{0} .
$$

We have to show the convergence on $\partial V_{1}$.

5. Let $F_{0}$ be the family of continuous $p$-forms $\varphi$ in $\bar{V}_{1}-B$, harmonic in $V_{1}-\bar{B}$, such that $\varphi=0$ on $\partial B$. The $q$-lemma for 0 -forms [4] has the following 
counterpart:

There exists a constant $q$ with $0<q<1$ such that

$$
\max _{\partial V_{0}}|\varphi| \leq q \max _{\partial r_{1}^{\prime}}|\varphi|
$$

for all $\varphi \in F_{0}$.

For the proof take the function $\omega$ continuous in $\vec{V}_{1}-B$ and harmonic in $V_{1}-\bar{B}$ such that $\omega=0$ on $\partial B$ and $\omega=1$ on $\partial V_{1}$. Let

$$
q=\sqrt{\max _{\partial r_{0}} \omega} .
$$

By the maximum principle it is clear that $0<q<1$. This is the desired constant $q$. In fact. to see (14) we may clearly assume that $|\varphi|^{2}>0$ on $\partial V_{1}$, for otherwise $|\varphi| \equiv 0$. We may also assume that $\max _{\partial v_{1}}|\varphi|=1$ and have $|\varphi|^{2} \leq \omega$ in $\bar{V}_{1}-B$, and $|\varphi|^{2} \leq q^{2}$ on $\partial V_{0}$.

6. Fix a neighborhood $N$ of $\partial V_{1}$. Clearly $\left|\sigma_{0}\right|$ is bounded in $N:\left|\sigma_{0}\right| \leq M$, say. By (14),

$$
\max _{\partial r_{0}}\left|K_{0}\left(L K_{0}\right)^{n-1} \sigma_{0}\right| \leq q \max _{\partial V_{1}}\left|K_{0}\left(L K_{0}\right)^{n-1} \sigma_{0}\right|=q \max _{\partial V_{1}}\left|\left(L K_{0}\right)^{n-1} \sigma_{0}\right| .
$$

Clearly by $(\gamma)$,

$$
\left|\left(L K_{0}\right)^{n} \sigma_{0}\right| \leq \max _{\partial V_{0}}\left|K_{0}\left(L K_{0}\right)^{n-1} \sigma_{0}\right|
$$

on $V_{o}=V-\bar{V}_{0}$ and of course on $N$. Thus by (15) and (16)

$$
\max _{N}\left|\left(L K_{0}\right)^{n} \sigma_{0}\right| \leq q \max _{N}\left|\left(L K_{0}\right)^{n-1} \sigma_{0}\right|
$$

and we conclude that

$$
\max _{N}\left|\left(L K_{0}\right)^{n} \sigma_{0}\right| \leq q^{n} M .
$$

This completes the proof of our claim that $\partial V_{0}$ can be reduced to an arbitrarily small sphere.

7. In [3] we proved the existence of a harmonic form $\rho$ in a locally flat $V$ with bounded $|\rho-\sigma|$ in $V-V_{0}$. Although it is not the main purpose of the present paper to discuss this existence, we close by showing that it follows on a hyperbolic locally flat $V$.

By the above reduction we may assume that $V_{0}$ and $V_{1}$ are concrete balls 
(and $\sigma=0$ on $\partial V_{0}$ ). Instead of $L$ and $K_{0}$ we take $L$ and $K$, and by exactly the same procedure our problem is reduced to the proof of the uniform convergence of

$$
\varphi=\sum_{n=0}^{\infty}(L K)^{n} \sigma_{0}
$$

8. Again we need a $q$-lemma of another kind. Let $N$ be a neighborhood of $\partial V_{1}$ with compact closure such that $\bar{N} \cap \bar{V}_{0}=\phi$.

Let $\tilde{F}$ be the family of continuous $p$-forms $\varphi$ on $\bar{V}_{s}=V-V_{0}$, harmonic in $V-\bar{V}_{0}$, such that $L \varphi=\varphi$ on $\bar{V}_{o}$.

Lemma. There exists a constant $q$ such that $0<q<1$ and

$$
\max _{N}|\varphi| \leq q \max _{\partial r_{0}}|\varphi|
$$

for any $\varphi$ in $\mathfrak{F}$.

The important fact to be observed is that $V_{0}$ is connected and thus the harmonic measure $u_{\partial V_{0}}$ of $\partial V_{0}$ with respect to $V_{o}$ satisfies $0<u_{\partial r_{0}}<1$ in $V_{o}$. The remainder of the proof is similar to that of (14).

9. The convergence is established as follows. Clearly $\left|L K_{\sigma_{0}}\right|$ is bounded in $N,\left|L K \sigma_{0}\right| \leq M$, say. The norm $|K \varphi|$ attains its maximum on $\partial V_{1}$, and therefore

$$
\max _{\partial r_{0}}\left|K(L K)^{n-1} \sigma_{0}\right| \leq \max _{\partial r_{1}}\left|K(L K)^{n-1} \sigma_{0}\right| \leq \max _{N}\left|(L K)^{n-1} \sigma_{0}\right| .
$$

By (18),

$$
\max _{N}\left|(L K)^{n} \sigma_{0}\right| \leq q \max _{\partial r_{0}}\left|L K(L K)^{n-1} \sigma_{0}\right|=q \max _{\partial r_{0}}\left|K(L K)^{n-1} \sigma_{0}\right|,
$$

and by (19) and (20),

$$
\max _{N}\left|(L K)^{n} \sigma_{0}\right| \leq q \max _{N}\left|(L K)^{n-1} \sigma_{0}\right| .
$$

We conclude that

$$
\max _{N}\left|(L K)^{n} \sigma_{0}\right| \leq q^{n-1} M .
$$

This completes the proof.

\section{BIBLIOGRAPHY}

[1] G. Duff-D. Spencer, Harmonic tensors in Riemannian manifolds with boundary, Ann. of Math., 56 (1952), 128-156. 
[2] M. Nakai-L. Sario, Harmonic fields with given boundary behavior in Riemannian spaces, J. Analyse Math. (to appear).

[3] M. Nakai-L. Sario, Point norms in the construction of harmonic forms (to appear).

[4] L. Sario-M. Schiffer-M. Glasner, The span and principal functions in Riemannian spaces, J. Analyse Math. 15 (1965), 115-154.

Nagoya University

and

University of California, Los Angeles 